\title{
Epibulbar Nodular Fasciitis
}

\author{
David John Massop ${ }^{\mathrm{a}}$ Paul Adam Frederick ${ }^{\mathrm{b}}$ He J. Li ${ }^{\mathrm{b}}$ Amy Lin ${ }^{\mathrm{b}}$ \\ ${ }^{a}$ Creighton University School of Medicine, Omaha, Nebr., USA; ${ }^{b}$ Moran Eye Center, \\ University of Utah, Salt Lake City, Utah, USA
}

\section{Keywords}

Epibulbar Nodular fasciitis · Conjunctival lesion · Scleral lesion

\begin{abstract}
Purpose: To report a case of epibulbar nodular fasciitis in a 32-year-old female and provide context by reviewing the current literature. Results: Using excisional biopsy, the patient was successfully diagnosed and treated for epibulbar nodular fasciitis. Upon follow-up, there has been no recurrence, consistent with the typical course for nodular fasciitis. Conclusions: Epibulbar nodular fasciitis is a rare process that can be successfully treated by surgical resection. While two cases of trauma-associated epibulbar nodular fasciitis have been present in the literature, our case did not have such a history. The etiology of nodular fasciitis remains unclear.

(C) 2016 The Author(s)

Published by S. Karger AG, Basel
\end{abstract}

\section{Introduction}

Nodular fasciitis is a benign soft-tissue tumor, resulting from fibroblast proliferation. While benign, the tumor can be mistaken for malignancy. Proper identification is essential in helping direct appropriate treatment. A histopathologic specimen is required for definitive diagnosis. Although trauma has been suggested as a possible cause, there is no clear etiology. Nodular fasciitis occurs in a variety of anatomical locations in both adult and pediatric populations, without gender predilection. Ocular involvement is rare but documented, especially 
in the orbit and adnexa [1-4]. Epibulbar nodular fasciitis is extremely rare, with seven reported cases prior to ours [1,5-9]. We present the case of a 32-year-old female with epibulbar nodular fasciitis.

\section{Case Report}

A 32-year-old female was referred to a cornea clinic with a bump on the left eye. After noticing the bump approximately 2 months prior, she saw an ophthalmologist who referred her for specialty care. The patient denied a history of pain or discharge, but did complain of a subjective decrease in visual acuity of the left eye. The patient was previously healthy, with unremarkable general medical and ocular histories. She denied a history of trauma or foreign body. Over the past 2 months, the patient noticed the lesion changing shape and color, describing the lesion as initially elevated and white, but progressively becoming flatter and yellow, with increased vascularity.

On examination, visual acuities were 20/20 and 20/40. A probable explanation for the decrease in visual acuity of the left eye is that the lesion caused a disruption in proper tear film distribution, resulting in blurred vision. Unfortunately, refraction was not performed at this time, but was performed postoperatively. Slit-lamp examination of the right eye was within normal limits. Examination of the left eye revealed a $7 \times 5.3 \mathrm{~mm}$ oval yellow elevated conjunctival lesion, inferior to the cornea and extending up to the limbus. The left cornea was clear.

The patient was started on a steroid taper (prednisolone 1\% drops four times per day for 1 week, and then three times per day for 1 week) and instructed to follow up in 2 weeks. At the follow-up, no improvement of the lesion was noted, and the patient was still complaining of decreased visual acuity. Examination of the left eye again revealed an oval yellow elevated conjunctival lesion, measuring $5.8 \times 5.8 \mathrm{~mm}$, focused at the inferior limbus at 5:30 o'clock (fig. 1a, b). The overlying conjunctiva was noted to be injected with dilated vessels. The mass was nontender and nonmobile. The remainder of the left-eye examination and right-eye examination was within normal limits. Prednisolone was stopped, and the patient was scheduled for excisional biopsy.

The patient was taken to the operating room for removal of the lesion, requiring excisional biopsy with anterior sclerectomy. During surgery, the lesion was pinkish-yellowish in color and was quite hard in consistency. It separated easily from the overlying conjunctiva, but was very adherent to the sclera. There was not a clear plane distinguishing the lesion from normal sclera, so the lesion was debulked, while keeping the natural contour of the sclera intact. The conjunctiva was closed primarily over the scleral defect using interrupted 8-0 Vicryl sutures. The patient tolerated the procedure well. The specimen was sent to the pathology laboratory for evaluation.

Postoperatively, the patient was placed on topical moxifloxacin for 1 week and a topical steroid taper for 3 weeks. She was followed during the postoperative period without complication. At approximately 6 months postoperatively, her visual acuity using a Snellen chart was OD 20/25 and OS 20/30. Her refraction was OD: $-1.00+0.50 \times 03920 / 20$ and OS: -1.75 $+1.00 \times 11620 / 20$. She had no signs of recurrence. 


\section{Case Reports in Ophthalmology}

\section{Pathologic Evaluation}

The specimen was observed under light microscopy with H\&E stain (fig. 2). Normal topography of the epithelium, substantia propria, and superficial sclera were identified. Deep to the substantia propria, irregular fibrous connective tissue was identified. Interesting features include proliferating spindle fibroblasts within the dense connective tissue. The fibroblasts appear non-atypical, and no sign of dysplasia or malignancy was noted. Normal sclera was identified, and the lesion did not extend into the sclera. Scant inflammatory cells were noted on high-power fields. No sign of fluid extravasation was noted. Interestingly, no myxoid tissue was noted in this specimen, which would be typical of nodular fasciitis. Given the clinical history, nodular fasciitis is the diagnosis most consistent with the histological features.

\section{Discussion}

While the exact pathophysiologic mechanism of nodular fasciitis is unknown, some have hypothesized that it is a reactive process of the connective tissue when exposed to repeated trauma or inflammation [10]. Evidence to support this theory is questionable both in ocular and nonocular nodular fasciitis. Indeed, several recent case series in pediatric nodular fasciitis have shown minimal to no history of trauma [11-13]. Of these 23 reported cases, only 1 had a history of trauma and 1 had a history of localized infection.

Of the 7 published cases of epibulbar nodular fasciitis, the most recent 2 have a plausible history of repetitive trauma [8, 9]. In 2005, Stone and Chodosh [8] described a case of epibulbar nodular fasciitis in a patient with floppy eyelid secondary to vigorous rubbing. In 2015, McClintic et al. [9] reported a case of corneal nodular fasciitis in a patient who also had a history of floppy eyelid syndrome. McClintic et al. postulate chronic irritation as a possible predisposing factor, a form of microtrauma. Interestingly, however, our patient denied any history of 'macro' trauma, such as vigorous rubbing, and has no discernable medical conditions, such as floppy eyelid syndrome, to predispose her to this described microtrauma. Despite no obvious source, it is still plausible that the patient experienced subclinical microtrauma.

Nodular fasciitis is a benign nodular reactive proliferation of fibroblasts and vascular tissue usually arising within the fascia. In the eye, it usually manifests in the orbit, eyelid, or episclera. Grossly, the lesions tend to be between 0.5 and $1.5 \mathrm{~cm}$ in diameter and tend to be round or oval. The specimen is not encapsulated. The specimens tend to be sparsely cellular, with scant infiltration of lymphocytes and mononuclear cells. Gross samples are mostly made of spindle fibroblasts (non-atypical), myxoid ground substance, and vasculature. Mitotic figures may be identified, and it is important not to confuse this with a sarcoma. The clinical history typically details an isolated tender rapidly growing subcutaneous nodule, presenting within 1-3 weeks.

Our case was initially concerning for episcleritis, a common differential diagnosis. Episcleritis is a benign recurring condition that often presents as hyperemia, edema, and infiltration, which are all limited to the episcleral tissue.

Episcleritis can be classified as simple episcleritis or nodular episcleritis. Simple episcleritis is characterized by diffuse edema, redness caused by engorged episcleral vessels, and small gray deposits. Histologically, there is vascular dilatation and perivascular lymphocytic infiltration. Proteinaceous fluid extravasation in the connective tissue appears as a uniform 
staining patch under simple H\&E staining. These fluid collections are not to be confused with the myxoid ground substances found in nodular fasciitis. The engorged vessels retain the normal radial position.

Nodular episcleritis is characterized by localized redness and edema. It is associated with systemic diseases such as rheumatoid arthritis and infection. An intraepiscleral nodule can be observed underlying the sclera. Histologically, chronic nongranulomatous inflammation of lymphocytes, plasma cells, and edema can be observed in the episcleral tissue. Chronic granulomatous inflammation is rarely seen. The differential diagnosis includes orbital lymphoma and idiopathic orbital inflammatory disease (formerly inflammatory pseudotumor) [14].

General considerations in the differentiation of nodular fasciitis and episcleritis are as follows: (1) episcleritis tends to be a more lymphocytic reaction, whereas nodular fasciitis tends to be more of a fibrocytic reaction, and (2) presence of a myxoid background points more towards nodular fasciitis. Clinical history is important in making a diagnosis, i.e. the presence of systemic inflammatory illness increases the likelihood of nodular episcleritis.

Differentiating between nodular fasciitis and episcleritis is important because the treatments differ. For nodular fasciitis, conservative steroid treatment may be attempted first to shrink the growth. Ultimately, the standard of care treatment for nodular fasciitis is surgical excision. As in our case, with excision, patients do extremely well. In contrast, episcleritis is often managed conservatively. As it is not typically sight-threatening and is often self-limited, symptomatic relief is the goal of therapy. For example, topical lubricants and oral NSAIDS can alleviate the discomfort.

An admitted deficiency of the preoperative examination in this patient was the lack of a manifest refraction. Six months postoperatively, the patient's uncorrected visual acuity had improved from $20 / 40$ to $20 / 30$, and was $20 / 20$ with a myopic and astigmatic correction. It is likely that refractive error was responsible for at least part of the decrease in vision preoperatively, and it is also possible that an uneven tear film distribution may have contributed to the decreased vision.

In summary, we presented a case of epibulbar nodular fasciitis in a 32-year-old female. Pathological diagnosis and treatment were achieved via excision. Unlike the two previous reports of epibulbar nodular fasciitis, our case has no clear history of trauma. Admittedly, subclinical microtrauma could still explain the etiology of the mass in our patient. However, this case lends limited support to a trauma/irritation-based theory of etiology. The pathophysiologic mechanism of nodular fasciitis is poorly understood, and further research is necessary.

\section{Statement of Ethics}

Patient care was provided under high ethical standards, with proper informed consent.

\section{Disclosure Statement}

Supported in part by an Unrestricted Grant from Research to Prevent Blindness, Inc., New York, N.Y., to the Department of Ophthalmology and Visual Sciences, University of Utah. 


\section{References}

Font RL, Zimmerman LE: Nodular fasciitis of the eye and adnexa. Arch Ophthalmol 1966;75:475-481.

$>2$ month-old child. Ophthal Plast Reconstr Surg 2001;17:144-148.

-3 Compton CJ, Clack JD, Thompson MP, Lee HH, Nunery WR: Nodular fasciitis of the orbit. Ophthal Plast Reconstr Surg 2014, Epub ahead of print.

-4 Gupta D, Tailor TD, Keene CD, Rostomily RC, Jian-Amadi A: A case of nodular fasciitis causing compressive optic neuropathy. Ophthal Plast Reconstr Surg 2014;30:e47-e49.

-5 Tolls RE, Mohr S, Spencer WH: Benign nodular fasciitis originating in Tenon's capsule. Arch Ophthalmol 1966;75:482-483.

6 Ferry AP, Sherman SE: Nodular fasciitis of the conjunctiva apparently originating in the fascia bulbi (Tenon's capsule). Am J Ophthalmol 1974;78:514-517.

7 Holds JB, Mamalis N, Anderson RL: Nodular fasciitis presenting as rapidly enlarging episcleral mass in a 3 year-old. J Pediatr Ophthalmol Strabismus 1990;27:157-160.

8 Stone DU, Chodosh J: Epibulbar nodular fasciitis associated with floppy eyelids. Cornea 2005;24:361362.

9 McClintic J, Plesec T, Billings S, Singh AD: Nodular fasciitis involving the cornea: a case report and review of the literature. Cornea 2015;34:1168-1169.

10 Price, EB, Silliphant WM, Shuman R: Nodular fasciitis: a clinicopatholgic analysis of 65 cases. Am J Clin Pathol 1961;35:122-136.

11 Hseu A, Watters K, Perez-Atayde A, Silvera VM, Rahbar R: Pediatric nodular fasciitis in the head and neck evaluation and management. JAMA Otolaryngol Head Neck Surg 2015;14:54-59.

12 Pandian TK, Zeidan MM, Ibrahim KA, Moir CR, Ishitani MB, Zarroug AE: Nodular fasciitis in the pediatric population: a single center experience. J Pediatr Surg 2013;48:1486-1489.

13 Tomita S, Thompson K, Carver T, Vazquez WD: Nodular fasciitis: a sarcomatous impersonator. J Pediatr Surg 2009;44:E17-E19.

14 Yanoff M, Fine BS: Ocular Pathology: A Text and Atlas, ed 3. Philadelphia, Lippincott Company, 1989.
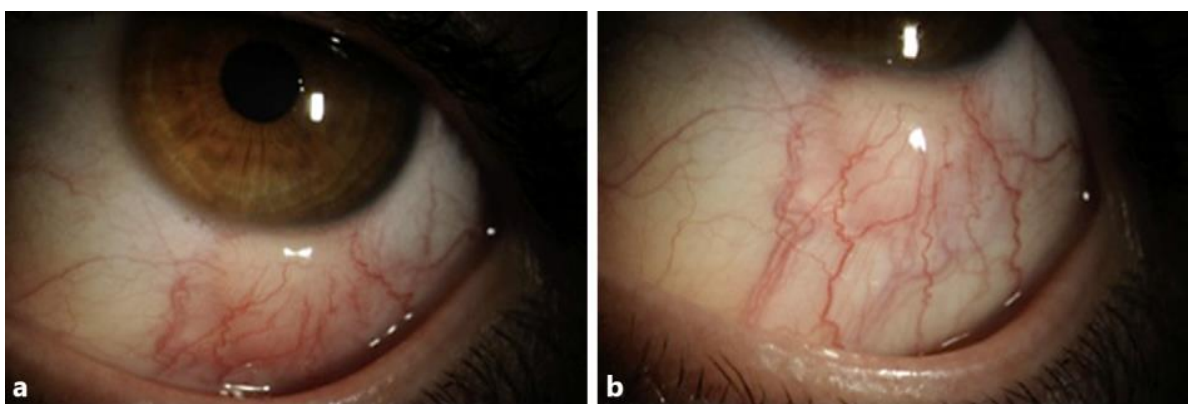

Fig. 1. a, b. Slit-lamp photos of the lesion with gentle traction on lower lid. a Left eye in primary position. b Left eye in superior gaze. 

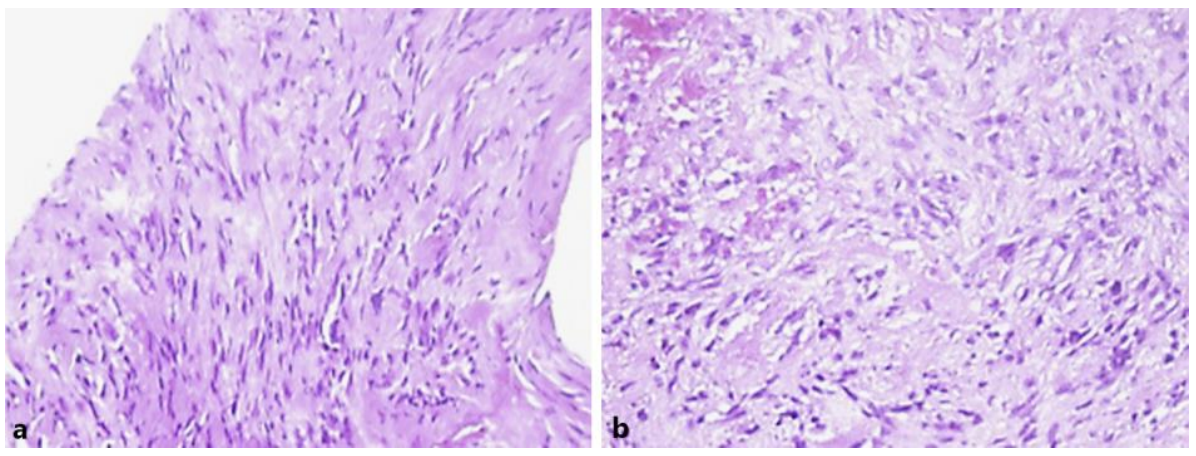

Fig. 2. a, b Pathology revealed a proliferation of plump and spindle fibroblasts, consistent with nodular fasciitis. a Dense fibrous tissue is observed which is representative of the samples. H\&E. $\times 20$. b Higher magnification of the sample reveals normal fibroblasts in a field of dense irregular fibrous tissue. H\&E. $\times 40$. 